\title{
Síndrome da apnéia obstrutiva do sono e doença cardiovascular
}

\author{
Obstructive sleep apnea and \\ cardiovascular disease
}

\begin{abstract}
Rodrigo P. Pedrosa1, Geraldo Lorenzi-Filho², Luciano F. Drager³
\end{abstract}
Pedrosa RP, Lorenzi-Filho G, Drager LF. Síndrome da apnéia obstrutiva do sono e doença cardiovascular. Rev Med (São Paulo). 2008 abr.-jun.;87(2):121-7.

RESUMO: A síndrome da apnéia obstrutiva do sono é uma entidade muito prevalente e ainda pouco diagnosticada entre os pacientes com cardiopatias. Caracteriza-se por episódios recorrentes de cessação do fluxo aéreo decorrente do colapso inspiratório das vias aéreas durante o sono, seguida de queda da saturação arterial de oxigênio. As alterações hemodinâmicas e metabólicas causadas por esses fenômenos que ocorrem centenas de vezes todas as noites predispõe ao desenvolvimento da hipertensão arterial, aterosclerose, acidente vascular cerebral, dentre outros. Discutiremos nessa breve revisão os princípios fisiopatológicos envolvidos na síndrome da apnéia obstrutiva do sono e sua interação com o sistema cardiovascular, como também as peculiaridades do seu tratamento com base nas mais recentes evidências clínicas.

DESCRITORES: Síndrome da apnéia do sono/epidemiologia. Doenças cardiovasculares. Arritmias cardíacas. Hipertensão. Condutas terapêuticas.

\section{INTRODUÇÃO}

A pnéia obstrutiva do sono é uma condição clínica muito comum e subdiagnosticada. Caracteriza-se por episódios recorrentes de cessação do fluxo aéreo decorrente do colapso inspiratório das vias aéreas durante o sono, seguida de queda da saturação arterial de oxigênio $^{34}$. Quando associada a sintomas diurnos, principalmente sonolência e/ou doença cardiovascular denomina-se síndrome da apnéia obstrutiva do sono (SAOS). Uma vez levantada a suspeita, deve-se optar pela realização da polissonografia noturna, considerada o exame de escolha para o diagnóstico. A

1. Médico Pós-Graduando do Laboratório do Sono, Disciplina de Pneumologia do InCor/FMUSP.

2. Diretor do Laboratório do Sono, Disciplina de Pneumologia do InCor / FMUSP, Disciplina de Cardiologia.

3. Médico Assistente da Unidade de Hipertensão do InCor/FMUSP.

Endereço para correspondência: Luciano F. Drager. Unidade de Hipertensão - Instituto do Coração (InCor), Universidade de São Paulo. Av. Dr. Enéas Carvalho de Aguiar, 44 - Bloco 2 - $2^{\circ}$ andar - sala 8. CEP 05403-904. São Paulo, Brasil. e-mail: luciano.drager@incor.usp.br 
polissonografia consiste na monitorização simultânea do eletroencefalograma, eletrooculograma, eletromiograma, saturação de oxigênio, fluxo de ar, esforço respiratório e freqüência cardíaca. A polissonografia noturna permite não só a avaliação da arquitetura e a eficiência do sono, mas também diagnosticar e diferenciar os distúrbios do sono. Diz-se que há hipopnéia quando há queda do fluxo inspiratório de mais de $30 \%$ associada à queda de saturação de oxigênio de mais de $4 \%$ por mais que 10 segundos e apnéia quando há queda de mais de $90 \%$ do fluxo pelo mesmo período. Classifica-se a SAOS de acordo com o número de apnéias e hipopnéias por hora de sono (IAH): leve de 5 a 14,9; moderada de 15 a 29 e grave quando maior ou igual a 30 .

O tratamento de escolha da SAOS consiste no uso da pressão positiva contínua nas vias aéreas (CPAP), que é um aparelho que fornece um fluxo de ar através de uma máscara facial nasal ou orofacial agindo como uma prótese pneumática para manter aberta a via aérea durante a inspiração e expiração ${ }^{1}$. Assim, promove remissão dos eventos respiratórios e consequentemente melhora a estrutura do sono e a qualidade de vida dos pacientes. No entanto, o CPAP também apresenta importante impacto cardiovascular.

\section{EPIDEMIOLOGIA}

Estudos epidemiológicos mostram que a SAOS acomete $4 \%$ dos homens e $2 \%$ das mulheres em uma população geral. Além do sexo masculino, obesidade, raça oriental, anormalidades estruturais de vias aéreas superiores, uso abusivo do álcool e história familiar são fatores de risco para a SAOS. Considerando apenas dados da polissonografia (desconsiderando os sintomas), sua prevalência chega a $24 \%$ em homens e $9 \%$ em mulheres ${ }^{38}$. A prevalência de SAOS em pacientes coronarianos é de $30 \%^{3}$; em portadores de fibrilação atrial, a SAOS está presente em cerca de 50\%; e na insuficiência cardíaca as estatísticas variam de 12 a 53\%. Particularmente na hipertensão arterial sistêmica, estudos mostram uma prevalência de SAOS em 35\% dos hipertensos, chegando a $70 \%$ em casos de hipertensão arterial refratária ${ }^{20}$. Já em obesos, a prevalência de SAOS chega a 40\%, enquanto $70 \%$ dos pacientes com SAOS possuem sobrepeso ou obesidade.

\section{SAOS E OBESIDADE}

Existe forte evidência que o excesso de peso é um fator causal para SAOS, mas dados demonstrando que a perda de peso reduz a intensidade da gravidade da SAOS ainda são escassos. Em uma análise longitudinal que avaliou 690 indivíduos em Wisconsin por um período de 4 anos, um incremento de $10 \%$ do peso corporal esteve associado com um incremento de 6 vezes no risco de SAOS ${ }^{27}$. Nesse mesmo estudo, redução de $10 \%$ do peso reduziu em $26 \%$ o índice de apnéia-hipopnéia. Outros estudos menores de perda de peso com tratamento cirúrgico ou dietético também demonstraram diminuição da SAOS $^{40}$. Ainda não se sabe o exato mecanismo da SAOS causada pela obesidade. A deposição de gordura nas vias aéreas pode ser um mecanismo na gênese da SAOS. Mudanças induzidas pela obesidade no mecanismo central do controle respiratório também podem estar implicadas ${ }^{36}$.

Enquanto a obesidade aumenta o risco de SAOS, a própria apnéia do sono pode predispor ao ganho de peso e obesidade. Pacientes com diagnóstico novo de SAOS apresentaram um ganho recente de peso no período que antecedeu o diagnóstico ${ }^{29}$. Somado a isso, o tratamento da SAOS com uso do CPAP por 6 meses demonstrou redução na gordura visceral de pacientes independentemente da associação com perda ponderal ${ }^{4}$. O mecanismo dessa associação é multifatorial. Pode estar relacionado aos hábitos de vida, como sedentarismo causado por sonolência diurna e capacidade física diminuída. Existem evidências de que o ganho de peso também pode estar associado a distúrbios no sistema endócrino. Nesse sentido, a leptina é uma substância produzida pelos adipócitos com função de supressão do apetite e aumento do gasto energético, contribuindo para a perda de peso. Indivíduos obesos têm altos níveis de leptina provavelmente por uma resistência a sua ação. Pacientes com SAOS possuem níveis plasmáticos $50 \%$ maiores de leptina comparados com controles obesos sem distúrbios respiratórios do sono ${ }^{30}$. Tudo isso parece estar intimamente relacionado num ciclo vicioso em que a obesidade predispõe ao aparecimento da SAOS, e essa se perpetua através da sua influência negativa sobre o peso corporal.

\section{HIPERTENSÃO ARTERIAL SISTÊMICA (HAS)}

A relação entre a SAOS e a HAS se deve, em grande parte, ao fato de a SAOS servir como um modelo de ativação simpática persistente, com diminuição na sensibilidade dos barorreceptores, hiperresponsividade vascular e alteração no metabolismo do sal e água que podem contribuir para a elevação da pressão arterial.

Atualmente, há dados suficientes na literatura para considerar a SAOS como uma causa secundária de $\mathrm{HAS}^{5}$. Muitas correlações iniciais vieram de estudos epidemiológicos, inicialmente com desenho do tipo caso-controle e posteriormente com a realização de coortes prospectivas com seguimento prolongado 
dos pacientes com SAOS. Em um destes estudos, 709 indivíduos que trabalhavam na Universidade de Wisconsin responderam a um questionário sobre hábitos de vida e história médica, além da aferição da pressão arterial, peso, altura e estudo polissonográfico. Realizou-se análise multivariada com ajuste para outros fatores de risco para doença cardiovascular, como tabagismo, obesidade, alcoolismo e pressão arterial presentes no início do estudo. Após seguimento de quatro anos mostrouse uma associação causal entre presença de SAOS na avaliação inicial e surgimento de HAS no seguimento, que foi independente de outros fatores de risco ${ }^{28}$. Adicionalmente os autores observaram uma relação dose-resposta entre o IAH e o risco de aparecimento da hipertensão arterial. Dados desta coorte detectaram um aumento de 3 vezes no risco de hipertensão durante o seguimento de 4 anos. Independente de outras doenças. Outras coortes que se seguiram mostraram resultados semelhantes aos descritos ${ }^{19,24}$.

Pacientes com SAOS apresentam repetitivos aumentos da pressão arterial associados aos episódios de apnéia e freqüentemente são considerados como "non-dippers" em decorrência de não haver uma queda apropriada da pressão arterial durante a noite. Este padrão é considerado de risco aumentado para o surgimento de doença cardiovascular.

Outro ponto importante é que a maioria dos pacientes hipertensos com SAOS não tratados tem níveis pressóricos elevados, a despeito do uso de muitas drogas anti-hipertensivas. Desta forma, em pacientes com hipertensão refratária ao tratamento, a SAOS pode se constituir em uma das principais causas de ausência de resposta ao tratamento antihipertensivo ${ }^{21}$.

Efeito do tratamento da apnéia no sono no controle da hipertensão

O tratamento da SAOS como coadjuvante no controle da pressão arterial foi abordado em diversos estudos. Bazzano et al. $^{2}$ em recente meta-análise demonstrou a redução da pressão arterial de 818 participantes em $2,46 \mathrm{mmHg}$ na pressão sistólica e 1,83 $\mathrm{mmHg}$ na pressão diastólica através do uso do CPAP. Entretanto, essa redução modesta da PA obtida nessa meta-análise é sujeita a críticas e pode não ser o único ou mesmo o maior efeito do uso de CPAP no sistema cardiovascular. A redução nas variações da pressão intratorácica e da pressão transmural miocárdica, além de diminuição da estimulação simpática sistêmica, podem ser de grande importância clínica. Além disso, essa análise englobou uma população muito heterogênea, incluindo um número significante de pacientes com HAS limítrofe ou mesmo normotensos, o que pode ter contribuído para redução modesta da pressão arterial ${ }^{8}$.

No que diz respeito ao tratamento da SAOS em pacientes com HAS refratária, as evidências são muito escassas. Logan et al. ${ }^{21}$ tratou apenas 11 pacientes com SAOS e HAS refratária com CPAP e demonstrou uma queda de $11 \mathrm{mmHg}$ na pressão arterial sistólica nas 24 horas. Martinez-Garcia et al. ${ }^{23}$ tratou a mesma população de hipertensos refratários de forma não randomizada por três meses e encontrou resultado similar, demonstrando queda da pressão sistólica de 5,2 $\mathrm{mmHg}$ nas 24 horas. Apesar de todas as suas limitações, estes estudos fornecem dados iniciais do impacto do tratamento da SAOS nesse subgrupo de pacientes de maior risco cardiovascular. Esses pacientes com HAS refratária podem ter o maior benefício em redução dos níveis tensionais em relação aos hipertensos de mais fácil controle participantes na maior parte dos estudos até o presente momento.

\section{ARRITMIAS}

A maioria das arritmias ocorre em pacientes com SAOS moderada a grave. A arritmia mais freqüentemente observada em pacientes com SAOS consiste na variação cíclica da freqüência cardíaca. Esta arritmia é caracterizada por bradicardia progressiva durante o período de apnéia com subseqüente taquicardia durante o período de retorno da respiração. A bradicardia geralmente começa com o início da apnéia com uma intensidade proporcional ao grau de hipoxemia e reflete uma mudança no tônus autonômico. Evidências mostram que cerca de $80 \%$ das bradicardias associadas à apnéia acontecem durante o sono REM, mostrando a vulnerabilidade do coração às influências autonômicas durante esta fase do sono. O mecanismo de taquicardia pósapnéia é provavelmente causado pela combinação do microdespertar e pela inibição do vago pelo reflexo de insuflação pulmonar resultando no aumento da freqüência cardíaca, diminuição da resistência vascular periférica e broncodilatação. A taquicardia não é sustentada provavelmente por retorno da influência parassimpática assim que a respiração retorna ${ }^{13}$.

As ectopias ventriculares têm sido relatadas de forma muito mais freqüente em pacientes com SAOS do que em pessoas sem esta condição clínica ${ }^{14}$, embora a incidência de taquicardia ventricular não sustentada seja semelhante ao da população geral. Por exemplo, num estudo enfocando pacientes portadores de cardiodesfibrilador implantável para a reversão de arritmias malignas, o número de choques realizados em pacientes com e sem SAOS 
foi semelhante ${ }^{11}$. Também é infreqüente a presença de potenciais tardios ventriculares, um fator de risco para o surgimento de arritmias malignas. Outras arritmias que têm sido relacionadas com a SAOS incluem a pausa sinusal e o bloqueio átrio-ventricular de $2^{\circ}$ grau, Mobitz II ${ }^{12}$

Evidências consistentes sugerem que pacientes com SAOS desenvolvem mais fibrilação atrial do que pessoas sem SAOS, independente de qualquer outro fator de risco, incluindo a obesidade ${ }^{10}$. Dentre os possíveis mecanismos responsáveis pela maior ocorrência de fibrilação atrial, destaca-se a evidência de remodelamento atrial promovido pela SAOS. Pacientes com SAOS, mesmo na ausência de hipertensão arterial, apresentam um aumento no tamanho atrial, conhecido fator que aumenta a predisposição para a ocorrência de arritmias $^{6}$.

\section{ATEROSCLEROSE, DOENÇA CORONARIANA E ACIDENTE VASCULAR CEREBRAL}

Há um interesse crescente na literatura da possível relação entre a SAOS, aterosclerose, doença coronariana e acidente vascular cerebral. Recentemente tem sido descrito na literatura que pacientes com SAOS apresentam uma aceleração no processo de aterosclerose. Em uma destas evidências, Drager et al. ${ }^{7}$ descreveram de forma pioneira a ocorrência de sinais precoces de aterosclerose (aumento da rigidez arterial, aumento da espessura íntima-média da carótida e do diâmetro da carótida) em pacientes aparentemente saudáveis com SAOS grave, isto é, que não apresentavam fatores de risco como hipertensão arterial, diabetes e tabagismo. Além disto, houve uma correlação entre a gravidade dos marcadores de aterosclerose com a gravidade da SAOS.

No que diz respeito à doença coronariana, até o momento as evidências são relativamente escassas e baseadas em uma relação de associação e não de uma relação causal. Estima-se que a prevalência de doença coronariana nos pacientes com SAOS esteja em torno de $25 \%$, sendo esta porcentagem mais alta nos pacientes com SAOS moderada e grave ${ }^{22}$. Já a prevalência da SAOS em pacientes com doença coronariana é estimada em $30 \%{ }^{16,32}$. A importância do reconhecimento da SAOS é respaldada por evidências sugerindo que a SAOS pode contribuir tanto para a progressão da doença coronariana, quanto para a instabilização de uma doença coronariana estabelecida. Neste sentido, estudos com registros simultâneos da polissonografia e do eletrocardiograma demonstraram a ocorrência de episódios de isquemia noturna em pacientes com SAOS, sendo mais comum durante o sono REM, fase onde os eventos respiratórios são mais comuns ${ }^{26}$. De forma interes- sante, o tratamento com o CPAP promoveu redução dos eventos isquêmicos noturnos.

Outro dado interessante é o que se refere à carga aterosclerótica coronariana. Em um estudo, o escore de Gensini, indicador da gravidade da aterosclerose coronariana, foi maior em apnéicos com eventos de dessaturação $>5$ eventos/hora de sono (evento de dessaturação definido como queda na saturação $\mathrm{O}_{2}>3 \%$ com duração de no mínimo 10 seg.) do que nos não dessaturadores ${ }^{15}$. Outro estudo que avaliou a relação entre a SAOS e a doença coronariana foi o Sleep Heart Health Study ${ }^{31}$, que revelou que a razão de chance para o desenvolvimento de insuficiência coronariana foi de 1,27 naqueles indivíduos com IAH $>11$ eventos/hora, sugerindo ser a SAOS um fator independente para a doença coronariana.

Com relação ao acidente vascular cerebral (AVC), estudos têm sugerido que a SAOS está associada à ocorrência de AVC e morte, sendo que a prevalência desta síndrome no AVC pode chegar a $60 \%{ }^{17,33}$ em comparação com 2 a $4 \%$ na população de meia idade. Poucos estudos, no entanto, avaliaram o papel da SAOS como fator de risco independente para estes desfechos após ajustar para outros fatores de risco. Yaggi et al. ${ }^{37}$ em estudo observacional de coorte incluiu 1022 pacientes dos quais 68\% apresentavam SAOS sendo o IAH > 5 e $32 \%$ eram os controles com $\mathrm{IAH}<5$ eventos/hora. A apnéia do sono foi independentemente associada à AVC e morte (OR: 2,24) após ajustar para os fatores confundidores (idade, sexo, raça, IMC, diabetes mellitus, hiperlipidemia, fibrilação atrial e HAS).

\section{SAOS E INSUFICIÊNCIA CARDÍACA CONGESTI-} VA (ICC)

A SAOS pode ser fator de risco para desenvolvimento de ICC. No Sleep Heart Study, que envolveu mais de 6000 homens e mulheres, evidenciou-se que a presença da SAOS aumentou em 2,38 vezes a probabilidade de um indivíduo ter ICC, independente de outros fatores de risco ${ }^{32}$. Um estudo recente mostrou que a presença da SAOS em pacientes com insuficiência cardíaca estabelecida está associada com um aumento da mortalidade no seguimento médio de três anos ${ }^{35}$. Como vimos anteriormente, o substrato fisiopatológico para o estabelecimento desta relação baseia-se no impacto da ativação simpática persistente em detrimento da inibição vagal do sistema cardiovascular em geral, bem como os efeitos deletérios do aumento da sobrecarga e da hipóxia sobre o miocárdio. Neste sentido, as conseqüências da ativação crônica do sistema simpático incluem a necrose do miócito, apoptose, downregulation e dessensibilização do receptor $\beta$-adrenérgico, 
Pedrosa RP et al. Síndrome da apnéia obstrutiva do sono e doença cardiovascular.

arritmogênese e aumento das taxas de mortalidade ${ }^{9}$. Estímulo nos nervos simpáticos do território renal promove a ativação do sistema renina-angiotensinaaldosterona, bem como a retenção de sódio e água. O comprometimento do barorreflexo e do controle da freqüência cardíaca são achados adicionais que cooperam para o aumento do risco de morte súbita nestes pacientes ${ }^{18}$. Em contraste com o que acontece em pacientes com ICC isolada, a presença conjunta da SAOS coopera para amplificar os distúrbios do controle cardiovascular também durante o sono. Portanto, torna-se uma combinação altamente desfavorável para gerar uma piora da função ventricular, surgimento de arritmias e por conseqüência a uma piora da sobrevida ${ }^{35}$.

\section{SAOS E MORTE SÚBITA}

O risco de eventos cardiovasculares na população geral é significativamente maior nas primeiras horas da manhã após o despertar (habitualmente entre às 6 horas da manhã e o meio-dia). Existem muitas explicações para justificar este padrão. O aumento dos eventos durante a manhã pode ser explicado em parte por mudanças no comportamento da atividade simpática, alterações circadianas do barorreflexo, aumento da coagulabilidade e de anormalidades eletrofisiológicas. Recentes evidências mostraram que o padrão circadiano de eventos cardiovasculares na SAOS é diferente da população em geral. Nesse sentido, Gami et al. ${ }^{10}$ avaliaram 112 pacientes com SAOS que tiveram quadro de morte súbita de origem cardíaca. Diferentemente da população em geral, estes autores observaram que pacientes com SAOS morrem mais no período da meia-noite às 6 horas da manhã do que no período da manhã. Além disso, esses autores observaram que, quanto mais grave a SAOS, maior a chance de o indivíduo ter morte súbita de madrugada do que em relação aos outros períodos. Esses dados sugerem que os eventos respiratórios durante a noite podem desencadear a ocorrência de doenças cardiovasculares, tais como o infarto agudo do miocárdio e o acidente vascular cerebral, em pacientes susceptíveis.

\section{CONSIDERAÇÕES FINAIS}

O pleno conhecimento da fisiopatologia da SAOS e suas múltiplas e variadas relações com o sistema cardiovascular contribuirão para o tratamento adequado de um número cada vez maior de pacientes portadores dessa síndrome, tendo em vista a "epidemia" de obesidade no mundo moderno. A busca ativa dessa síndrome precisa começar a ser realizada, a partir da incorporação de perguntas simples no interrogatório sintomatológico, como a presença de ronco e sonolência diurna, a fim da suspeição diagnóstica e tratamento efetivo.

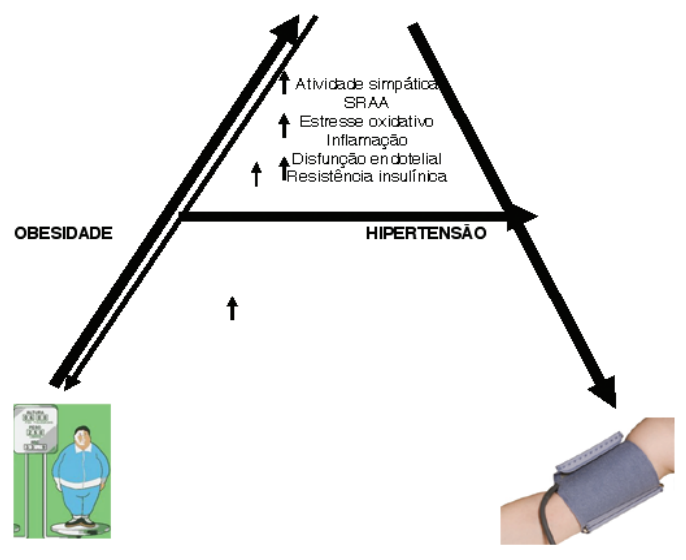

Figura 1. Relação causal entre SAOS (Síndrome de apnéia obstrutiva do sono), hipertensão arterial e obesidade. (SRAA: Sistema renina-angiotensina-aldosterona)

Pedrosa RP, Lorenzi-Filho G, Drager LF. Obstructive sleep apnea and cardiovascular disease. Rev Med (São Paulo). 2008 abr.-jun.;87(2):121-7.

\begin{abstract}
Obstructive sleep apnea is a very prevalent syndrome that is commonly underdiagnosed in patients with heart disease. It is characterized by recurrent episodes of cessation of respiratory airflow caused by upper airway inspiratory collapse during sleep, with a consequent decrease in oxygen saturation. Hemodinamic and metabolic disturbances caused by this phenomenon that occurs hundred of time during the night contribute to the onset of hypertension, atherosclerosis and stroke. We will discuss in this brief update physiopathological principles involved in obstructive sleep apnea syndrome and its interactions with the cardiovascular system, as well as peculiarities in the treatment of this syndrome based on the most recent clinical evidence.
\end{abstract}

KEY WORDS: Sleep apnea syndromes/epidemilogy. Cardiovascular diseases. Arrythmias, cadiac. Hypertension. Therapeutical approaches. 


\section{REFERÊNCIAS}

1. Basner RC. Continuous positive airway pressure for obstructive sleep apnea. N Engl J Med. 2007;356:1751-8.

2. Bazzano LA, Khan Z, Reynolds K, He J. Effect of nocturnal nasal continuous positive airway pressure on blood pressure in obstructive sleep apnea. Hypertension. 2007;50:417-23.

3. Bhama JK, Spagnolo S, Alexander EP, Greenberg M, Trachiotis GD. Coronary revascularization in patients with obstructive sleep apnea syndrome. Heart Surg Forum. 2006;9(6):E813-7.

4. Chin K, Shimizu K, Nakamura T, Narai N, Masuzaki $\mathrm{H}$, Ogawa $\mathrm{Y}$, et al. Changes in intra-abdominal visceral fat and serum leptin levels in patients with obstructive sleep apnea syndrome following nasal continuous positive airway pressure therapy. Circulation. 1999;100:706-12.

5. Chobanian AV, Bakris GL, Black HR, Cushman WC, Green LA, Izzo JL Jr, et al. Seventh report of the Joint National Committee on Prevention, Detection, Evaluation, and Treatment of High Blood Pressure. Hypertension. 2003;42(6):1206-52.

6. Drager LF, Bortolotto LA, Figueiredo AC, Silva BC, Krieger EM, Lorenzi-Filho G. Obstructive sleep apnea, hypertension and their interaction on arterial stiffness and heart remodeling. Chest. 2007;131:1379-86.

7. Drager LF, Bortolotto LA, Lorenzi MC, Figueiredo AC, Krieger EM, Lorenzi-Filho G. Early signs of atherosclerosis in obstructive sleep apnea. Am J Respir Crit Care Med. 2005;172(5):613-8.

8. Floras JS, Bradley TD. Treating obstructive sleep apnea: is there more to the story than 2 millimeters of mercury?. Hypertension. 2007;50:289-91.

9. Floras JS. Clinical aspects of sympathetic activation and parasympathetic withdrawal in heart failure. $\mathrm{J} \mathrm{Am}$ Coll Cardiol. 1993;22(4 Suppl A):72A-84A.

10. Gami AS, Hodge DO, Herges RM, Olson EJ, Nykodym $\mathrm{J}$, Kara T, et al. Obstructive sleep apnea, obesity, and the risk of incident atrial fibrillation. J Am Coll Cardiol. 2007;49(5):565-71.

11. Grimm W, Koehler U, Fus E, Hoffmann J, Menz V, Funck R, et al. Outcome of patients with sleep apneaassociated severe bradyarrhythmias after continuous positive airway pressure therapy. Am $\mathrm{J}$ Cardiol. 2000;86(6):688-92, A9.

12. Guilleminault $\mathrm{C}$, Connolly SJ, Winkle RA. Cardiac arrhythmia and conduction disturbances during sleep in 400 patients with sleep apnea syndrome. Am J Cardiol. 1983;52(5):490-4.

13. Gula LJ, Krahn AD, Skanes AC, Yee R, Klein GJ. Clinical relevance of arrhythmias during sleep: guidance for clinicians. Heart. 2004;90(3):347-52.

14. Harbison J, O'Reilly P, McNicholas WT. Cardiac rhythm disturbances in the obstructive sleep apnea syndrome: effects of nasal continuous positive airway pressure therapy. Chest. 2000;118(3):591-5.

15. Hayashi M, Fujimoto K, Urushibata K, Uchikawa S, Imamura H, Kubo K. Nocturnal oxygen desaturation correlates with the severity of coronary atherosclerosis in coronary artery disease. Chest. 2003;124(3):936-41.

16. Hung J, Whitford EG, Parsons RW, Hillman DR. Association of sleep apnoea with myocardial infarction in men. Lancet. 1990;336(8710):261-4.

17. Kryger MH. Pathophysiology of obstructive sleep apnea syndrome. In: Fabiani M, editor. Surgery for snoring and obstructive sleep apnea syndrome. The Netherlands: Kugler;2003. p.47-61.

18. La Rovere MT, Pinna GD, Hohnloser SH, Marcus FI, Mortara A, Nohara R, et al. Autonomic tone and reflexes after myocardial infarcton. Baroreflex sensitivity and heart rate variability in the identification of patients at risk for life-threatening arrhythmias: implications for clinical trials. Circulation. 2001;103(16):2072-7.

19. Lavie P, Herer P, Hoffstein V. Obstructive sleep apnoea syndrome as a risk factor for hypertension: population study. BMJ. 2000;320(7233):479-82.

20. Logan AG, Perlikowski SM, Mente A, Tisler A, Tkacova $R$, Niroumand $M$, et al. High prevalence of unrecognized sleep apnoea in drug-resistant hypertension. J Hypertens. 2001;19(12):2271-7.

21. Logan AG, Tkacova R, Perlikowski SM, et al. Refractory hypertension and sleep apnoea: effect of CPAP on blood pressure and baroreflex. Eur Respir J. 2003;21:241-7.

22. Maekawa M, Shiomi T, Usui K, Sasanabe R, Kobayashi T. Prevalence of ischemic heart disease among patients with sleep apnea syndrome. Psychiatry Clin Neurosci. 1998;52(2):219-20.

23. Martínez-García MA, Gómez-Aldaraví R, SolerCataluña JJ, Martínez TG, Bernácer-Alpera B, RománSánchez P. Positive effect of CPAP treatment on the control of difficult-to-treat hypertension. Eur Respir J. 2007;29:951-7.

24. Nieto FJ, Young TB, Lind BK, Shahar E, Samet JM, Redline $\mathrm{S}$, et al. Association of sleep-disordered breathing, sleep apnea, and hypertension in a large community-based study. Sleep Heart Health Study. JAMA. 2000;283(14):1829-36.

25. Martha R Shepertycky MR, Al-Barrak M, Kryger $\mathrm{MH}$. Obstructive sleep apnea syndrome. 1. Effect of treatment on cardiovascular morbidity. Sleep Biol Rhythms. 2003;1(1):15-28.

26. Peled N, Abinader EG, Pillar G, Sharif D, Lavie P. Nocturnal ischemic events in patients with obstructive sleep apnea syndrome and ischemic heart disease: effects of continuous positive air pressure treatment. J Am Coll Cardiol. 1999;34(6):1744-9.

27. Peppard PE, Young T, Palta M, Dempsey J, Skatrud J. Longitudinal study of moderate weight change and sleep 
Pedrosa RP et al. Síndrome da apnéia obstrutiva do sono e doença cardiovascular.

disordered breathing. JAMA. 2000;284:3015-21.

28. Peppard PE, Young T, Palta M, Skatrud J. Prospective study of the association between sleep-disordered breathing and hypertension. N Engl J Med. 2000;342(19):1378-84.

29. Phillips BG, Hisel TM, Kato M, Pesek CA, Dyken ME, Narkiewicz K, et al. Recent weight gain in patients with newly diagnosed obstructive sleep apnea. J Hypertens. 1999;17:1297-300.

30. Phillips BG, Kato M, Narkiewicz K, Choe I, Somers VK. Increases in leptin levels, sympathetic drive, and weight gain in obstructive sleep apnea. Am J Physiol. 2000;279:H234-7.

31. Schafer H, Koehler U, Ewig S, Hasper E, Tasci $\mathrm{S}$, Luderitz B. Obstructive sleep apnea as a risk marker in coronary artery disease. Cardiology. 1999;92(2):79-84.

32. Shahar E, Whitney CW, Redline S, Lee ET, Newman $A B$, Javier Nieto $F$, et al. Sleep-disordered breathing and cardiovascular disease: cross-sectional results of the Sleep Heart Health Study. Am J Respir Crit Care Med. 2001;163(1):19-25.

33. Shepertycky MR, Al-Barrak M, Kryger MH. Morbidity and mortality in obstructive sleep apnea syndrome. 1. Effect of treatment on cardiovascular morbidity. Sleep
Biol Rhythms. 2003;1(1):15-28.

34. Sleep-related breathing disorders in adults: recommendations for syndrome definition and measurement techniques in clinical research. The Report of an American Academy of Sleep Medicine Task Force. Sleep. 1999; 22:667-89.

35. Wang H, Parker JD, Newton GE, Floras JS, Mak S, Chiu KL, et al. Influence of obstructive sleep apnea on mortality in patients with heart failure. J Am Coll Cardiol. 2007;49(15):1625-31.

36. Wolk R, Shamsuzzaman ASM, Somers VK. Obesity, sleep apnea, and hypertension. Hypertension. 2003;42:1067-74.

37. Yaggi HK, Concato J, Kernan WN, Lichtman JH, Brass LM, Mohsenin V. Obstructive sleep apnea as a risk factor for stroke and death. $\mathrm{N}$ Engl $\mathrm{J}$ Med. 2005;353(19):2034-41.

38. Young T, Palta M, Dempsey J, Skatrud J, Weber S, Badr S. The occurrence of sleep-disordered breathing among middle-aged adults. $\mathrm{N}$ Engl $\mathrm{J}$ Med. 1993;328(17):1230-5.

39. Young T, Peppard PE, Gottlieb DJ. Epidemiology of obstructive sleep apnea. Am J Respir Crit Care Med. 2002;165:1217-39. 